\title{
La capacidad condicional de resistencia: Su relación con la selección de talentos deportivos en la ESPOCH
}

DOI: https://doi.org/10.33262/ap.v3i3.1.98

(c) (1) () ()

The conditional resistance capacity and its relationship with the selection of sports talent at ESPOCH.

\author{
Olguer Fabián Sánchez Espinoza. ${ }^{1}$, Luis Gustavo Díaz. ${ }^{2}$ \& Jorge Giovanny Tocto \\ Lobato. $^{3}$
}

\begin{abstract}
Introduction. Evaluating the physical condition of resistance in university students through Test or physical tests, for the detection of sports talents, constitutes a relevant tool. Objective. Incorporate a sports talent detection system, from the approach of timely diagnosis, through University Physical Education Programs, which allow controlling and classifying physical capacities, taking into account the individual characteristics of each student. Methodology. The research followed a mixed, descriptive approach using theoretical and empirical methods. The sample consisted of 1098 students, fulfilling the inclusion criteria of studying at the Higher Polytechnic School of Chimborazo (ESPOCH) in its 7 faculties. A single test or physical test was used, which provided the necessary results for the selection of students with sports talent. Results. 1. An essential indicator for measuring the performance of physical endurance capacity is provided, facilitating knowledge regarding the number of students who may have the primary conditions to be considered possible sports talents. 2. The importance and usefulness of timely diagnosis is reaffirmed, through the use of physical tests to measure physical condition and facilitate the detection of students who constitute potential sports potentials. Conclusions. Among the aspects most evaluated in the talent selection process in terms of endurance capacity are genetic factors such as: VO2 max, heart rate, beats per minute and respiratory rate. It
\end{abstract}

1 Gobierno Autónomo Descentralizado Municipal de Mocha., Chimborazo, fsanchezec@yahoo.com, https://orcid.org/0000-0001-9130-4429

2 Escuela Superior Politécnica de Chimborazo ESPOCH, gustavodiaz@espoch.edu.ec, https://orcid.org/0000-0003-2615-5003

3 Escuela Superior Politécnica de Chimborazo ESPOCH, jtocto@qespoch.edu.ec, https://orcid.org/00000002-1759-1316 
is shown that it is essential to consider sports selection as a process in which the largest number of practitioners must necessarily be involved, in such a way that it allows the evaluation tests to be applied to the generality, the larger the sample, the greater the number possible to be selected for the practice of a certain sport.

Keywords: physical endurance capacity, talent, sports team, tests physical.

\section{Resumen}

Introducción. Evaluar la condición física de resistencia en los estudiantes universitarios a través de test o pruebas físicas, para la detección de talentos deportivos, constituye una herramienta relevante. Objetivo. Incorporar un sistema de detección de talentos deportivos, desde el enfoque del diagnóstico oportuno, mediante programas de educación física universitarias, que permitan controlar y clasificar las capacidades físicas, teniendo en cuenta las características individuales de cada estudiante. Metodología. La investigación siguió un enfoque mixto, de tipo descriptivo empleando métodos teóricos y empíricos. La muestra estuvo constituida por 1098 estudiantes, cumpliendo el criterio de inclusión de cursar estudios en la Escuela Superior Politécnica de Chimborazo (ESPOCH) en sus 7 facultades. Se trabajó con un único Test o prueba física, que aportó los resultados necesarios para la selección de los estudiantes con talento deportivo. Resultados. 1. Se aporta un indicador esencial de medición del desempeño de la capacidad física de resistencia, facilitando el conocimiento respecto a la cantidad de estudiantes que pudieran tener las condiciones primarias para ser considerados posibles talentos deportivos. 2. Se reafirma la importancia y utilidad del diagnóstico oportuno, mediante la utilización de Test físicos para medir la condición física y facilitar la detección de los estudiantes que constituyen posibles potenciales deportivos. Conclusiones. Entre los aspectos más evaluados en el proceso de selección de talentos en cuanto a la capacidad de resistencia están los factores genéticos tales como: el VO2 máx., la frecuencia Cardiaca, las pulsaciones por minutos y la frecuencia respiratoria. Se demuestra que es esencial considerar la selección deportiva como un proceso en el que necesariamente deben involucrarse la mayor cantidad de practicantes, de forma que permita aplicar los Test de evaluación a la generalidad, cuanto mayor sea la muestra, mayor será la cantidad posibles a ser seleccionados para la práctica de un determinado deporte.

Palabras clave: capacidad física de resistencia, talento, selección deportiva, pruebas físicas.

\section{Introducción}

Ante todo, es importante señalar que los estudiantes universitarios se encuentran en una etapa de su vida en la ya han concluido su proceso de maduración biológica con su respectivo desarrollo psicomotriz. Al respecto autores como Chacón et al. (2017), la definen como una fase muy compleja para los jóvenes a la que denomina adultez 
emergente, por otra parte, otros autores como Arnett (2014), definen a este periodo como la última fase de la adolescencia, en la que, conforme a Castro (2016) y O'Connor et al. (2011), aunque ya se han originado todos los cambios biológicos y físicos inherentes a esta etapa, aún falta por instalar en su totalidad las características socio-afectivas y culturales propias de la adultez.

Estudios realizados por diversos autores, entre los que destacan Van Dyck, et al. (2015), han demostrado que la etapa universitaria se puede catalogar como un periodo crítico en la adquisición de hábitos de vida saludables en los estudiantes universitarios, dado que los mismos transitan de una rutina habitual de realización de actividad física en su etapa infantil y de adolescencia, a una merma significativa del nivel de este tipo de actividad, con el correspondiente incremento de los hábitos de sedentarismo

En esta dirección es importante se señalar que se ha demostrado que en el periodo de transición de la adolescencia a la adultez se origina un descenso significativo de los niveles de actividad física, lo que ha sido señalado por varios autores tales como Han et al. (2008) y Raustorp \& Ekroth (2013). De igual manera al respecto se han pronunciado Cocca, et al. (2014), lo cual contradice lo estipulado por la OMS (2010) sobre la buena salud en esta etapa de vida, en la que se recomienda que los adultos de 18 a 64 años practiquen al menos 30 minutos diarios de AF moderada o vigorosa (AFMV).

Relacionado con ello, se han desarrollado distintos estudios por diferentes autores, entre los que se encuentran Práxedes et al. (2016), quienes al respecto señalan que el bajo grado de cumplimiento de las recomendaciones de la OMS en este periodo, se debe al cambio de estilo de vida que se origina en la transición de la Educación Secundaria a la universidad. Este hecho según Engberg et al. (2012) y Keller, et al. (2007), puede dar origen en los estudiantes nuevos hábitos y la reconstrucción de su tiempo de ocio, en detrimento del tiempo de actividad física.

En relación a ello, diversos autores, como Martínez, et al. (2012), suponen que la no continuidad de las clases de Educación Física, que representa una asignatura con carácter obligatorio en la etapa de Educación Secundaria, puede originar en los estudiantes universitarios una disminución de los niveles de práctica de la actividad física.

En referencia a lo anterior, estudios realizados por Rodríguez, et al. (2018), han determinado las diferencias que existen en los niveles de actividad física y del gasto energético en los estudiantes universitarios de a medida que transcurre el tiempo de la vida universitaria, observando que los valores referentes a ambas variables parecen descender con el paso de los años, pero no de manera significativa.

Es por esto primordial, la atención que se requiere por parte de las instituciones universitarias para revertir esta situación. Al respecto autores como Aránguiz, et al. (2010), señalan la importancia que reviste que las autoridades universitarias consideren incluir las actividades físico-deportivas en la malla curricular semestral como un requisito para la culminación de la carrera, tal como se aplica en algunos países como Japón y Cuba. 
De ahí que el aspecto relacionado con la condición física de los estudiantes universitarios, ha devenido en un tema de marcada relevancia por su vinculación con la realidad social, relacionado a una buena salud, mejor calidad de vida y de ocio, así como por la práctica de la propia actividad física relacionada con la actividad deportiva, la que requiere al menos de un desarrollo aceptable de las Capacidades Físicas Básicas y de las Cualidades Motrices de los mismos.

Al respecto estudios realizados por Chacón, et al. (2018), han observado que las personas que practican actividades físicas o deportes al menos tres horas a la semana, obtienen resultados más elevados comparados con los que no lo realizan, en los parámetros de masa magra (aumento de la masa de los músculos sin aumentar la grasa) y en el volumen consumido de oxígeno mientras se realiza un minuto de ejercicio físico intenso (VO2 $\max$.$) .$

Las Capacidades Físicas Básicas están constituidas por la fuerza, la resistencia, la flexibilidad y la velocidad, las cuales pueden ser mejoradas mediante la practica periódica del ejercicio físico, preferiblemente que se encuentren recogidos en programas de Educación Física, que hayan sido elaborados teniendo en cuenta las particularidades de los estudiantes universitarios.

Este aspecto es fundamental y ha sido destacado por autores, como Sáez \& Gutiérrez (2007) y Martínez (2001), señalando la importancia de organizar los contenidos de la Educación Física referidos a la mejora de las cualidades físicas, ya sea las condicionales como las perceptivo motrices.

Por otra parte, Ortega at al (2005), Carreras y Ordóñez (2007) \& García, et al. (2007), han resaltado que las acciones de mejoras de las capacidades condicionales, específicamente la resistencia aeróbica es imprescindible para el mantenimiento de los niveles óptimos de actividad física, constituyendo además una cuestión de salud pública.

En esta dirección, según Vinuesa \& Vinuesa (2016), definen la capacidad física de resistencia, como el conjunto de capacidades físicas y psíquicas que permiten a la persona la prolongación del esfuerzo con eficacia, haciendo retrasar o soportar la fatiga con la consiguiente recuperación, de manera rápida, de los esfuerzos realizados con antelación.

De igual forma, Weineck, (2005), identifico la capacidad física de resistencia, como la capacidad del deportista para soportar la fatiga psicofísica.

Autores como Chaparro, et al. (2019) han señalado que la resistencia constituye una de las capacidades físicas más importantes del ser humano, implicando que contar con un buen estado de resistencia depara disimiles beneficios tales como: aumenta el tiempo de esfuerzo, mejora el ritmo del ejercicio, reduce el tiempo necesario para la recuperación, reduce el cansancio y la sensación de fatiga, mejora la capacidad respiratoria, disminuye las pulsaciones tanto en activo como en estado de reposo, contribuye con la perdida de grasa corporal y mejora el metabolismo. 
Respecto a la capacidad de resistencia física, Jaimes, et al (2019), la han denominado como la capacidad psicofísica de una persona para soportar la fatiga ante un ejercicio de cierta intensidad y/o duración, así como la capacidad de recuperación después de ejecutado. La misma está conformada por varios parámetros evaluativos entre los que podemos citar la Resistencia aeróbica y la Resistencia anaeróbica.

Conforme a Shephard, \& Astrand (2007), la Resistencia en función de la vía energética utilizada, se clasifica en Resistencia anaeróbica, o sea, consta de un trabajo de esfuerzo corto (10-60 segundos) y Resistencia anaeróbica, o sea, trabajo de esfuerzo largo (mayor a 3 minutos).

Diferentes autores, entre los que podemos citar a Alfonso (2017), han señalado que la capacidad de resistencia física presenta una importancia primordial, dado que la misma se corresponde con la capacidad de mantener un esfuerzo sostenido en el tiempo, e independientemente a que este sea aeróbico o anaeróbico, resulta imprescindible para el óptimo desarrollo del potencial físico del individuo, en el que el entrenamiento anaeróbico propicia el desarrollo de la masa muscular y el entrenamiento aeróbico contribuye al desarrollo de resistencia propiamente.

En relación a esta capacidad física, algunos autores entre los que podemos citar a Vaca et al (2017), han expresado que para alcanzar un óptimo resultado de trabajo en la resistencia debemos obtener un equilibrio entre el oxígeno que se toma y el que se consume por el organismo, teniendo en cuenta que, dependiendo de la persona, oscila entre 120 y 130 pulsaciones por minuto.

En este sentido, Guimaraes (2002), han remarcado lo importante de entrenar la resistencia, dado que produce mejora cardiovascular, es decir, da lugar a que el bombeo de la sangre sea mayor y con un número menor de latidos; produce mejora de la resistencia de los músculos para la realización de un esfuerzo intenso; provoca una disminución de pulsaciones en reposo, produce una mejora de la capacidad pulmonar, así como produce una mejora del metabolismo.

Por lo anteriormente expuesto, Delgado et al (2007), expresaron que la capacidad física de resistencia, es una capacidad que debe ejercitarse siempre antes de iniciarse el desarrollo de actividades de larga duración en las que la potencia y la fuerza marcan un rol importante, dado que, si no se cuenta con una buena preparación para resistir, será muy difícil poderse sostener durante todo el tiempo de duración de la actividad. Ello implica que es primordial desarrollar la resistencia para contar con una base física.

Es por ello y en correspondencia con Zea, et al. (2018), que los programas de Educación Física Universitaria necesariamente deben ser capaz de controlar y clasificar las capacidades físicas, teniendo en cuenta la delimitación de los alcances y las limitaciones de cada estudiante universitario.

En este sentido y de acuerdo a Morales \& González (2014), seguido de Torres y otros autores en el (2017) se plantea que constituye precisamente uno de los objetivos 
primordiales de estos programas, realizar la propuesta justificada de como deberán ser planificadas las cargas físicas y cuál sería la proyección al diseñar e implementar un programa curricular especializado de Educación Física, adaptado a las necesidades y características de los estudiantes.

En referencia al talento deportivo diversos autores lo han conceptualizado y a su vez se ha modificado a través del tiempo. Entre los conceptos más actualizados podemos citar a Jurgen Weineck (2005, p 111) quien al respecto señala que el talento deportivo, comprende al conjunto de situaciones deportivas y el desarrollo que en ella se genera, por un adolescente o atleta, determinada por las cualidades personales y del proceso de actividad motora del individuo. Y agrega que, en vista a ello, la aptitud del individuo debe considerarse como un resultado de la confrontación activa de la personalidad del atleta con el contexto en que se desenvuelve.

Paucar (2016), han resumido al talento deportivo como un individuo predispuesto a un deporte, el cual se encuentra en un proceso evolutivo dentro de entorno ambiental y social, cuya combinación determinará la valoración final del futuro deportista. Como plantea el propio autor, estos individuos cuentan con desempeños deportivos y características físicas, superiores a la media normal en el desarrollo de una cualidad o capacidad específica, con una predisposición genética o de actitud, que los hace ser diferentes en el marco de un grupo o sociedad, siempre que sean adecuadamente identificados los estadios del desarrollo físico y motor de la población estudiada, por medio de los diferentes instrumentos de medición de las variables antropométricas y su clasificación.

En relación a lo anterior Abbot \& Collins (2004) ya habían señalado que el concepto de talento constituye una definición dinámica dado que no solo en el influyen diversos factores de rendimiento, sino además que evoluciona a lo largo del tiempo, debido a ello los modelos predictivos de detección de talentos que se basan en los perfiles de los deportistas presentan una reducida probabilidad de alcanzar el éxito.

Por otra parte, Simonton (1999), ha remarcado que como el talento es multidimensional, los individuos pueden remediar las debilidades que pudieran tener en un componente del deporte que practican, con fortalezas que tienen en otros determinados factores, a lo que denominan “el fenómeno de la compensación”.

En este sentido y en concordancia con Calvo, et al. (2013), el dominio en una actividad deportiva, no estará determinado por una elevada especialización en un único componente, al contrario, será por la integración de los diferentes factores de rendimiento, lo cual demuestra las diferencias que trascienden entre deportistas de la misma especialidad, en relación a su rendimiento.

Ligado a esto debemos señalar la importancia que reviste la aplicación de test o pruebas físicas, que serán las que aportaran la información necesaria que permitirán realmente evaluar el estado físico y de rendimiento del individuo y constatar su nivel físico en relación al grupo. No obstante, el test físico no basta para poder contar con un resultado óptimo, si no se realiza teniendo en cuenta el principio de transferencia, o sea la influencia 
que puede tener un ejercicio o acción motriz sobre el rendimiento de otra acción motriz distinta, o sea deben realmente ser estimulados los diversos factores del rendimiento en la actividad que recepta la transferencia, como pudieran ser el ángulo en que se aplica la fuerza, los tipos de activación muscular, las fases del movimiento y otros, dentro del entrenamiento.

En relación a lo expuesto, Martínez (2002, p.29), se ha referido a la aplicación de los test físicos, acotando que lo anterior es cierto no debiéndose olvidar que la condición física como tal, tiene incluido varios principios como el de transferencia, por lo que la realización de test o pruebas no serán efectivos si el mismo no prevé una proyección o transferencia hacia otros aspectos del entrenamiento de la actividad deportiva y de la propia cotidianidad del individuo. Ello constata que la condición física no solo se determina mediante los resultados que arroje la observación de la aplicación de pruebas o test físicos de un individuo, sino que se hace imprescindible tener en cuenta estos detalles y su relación con la cotidianidad del sujeto de estudio.

Domínguez y Duvergel (2015), han expresado que la propuesta de test para la selección de talento reviste una importancia primordial para los entrenadores y profesores de Educación Física, debido a que prescindirá la problemática de los criterios, aplicándose un mediante un método científico, lo cual elimina los errores que se cometen al seleccionar individuos que realmente no cuentan con las características propias que pudiera catalogarse como un talento, llegando incluso a no promover verdaderos prospectos que si reúnen las condiciones.

En esta línea un aspecto que viene a locación es el referido a la selección deportiva, la cual según Matvevv 2001, (p. 102), expresa que no existe una interpretación unificada sobre su noción, relacionándola la mayoría de los expertos con la previa predisposición del individuo relacionado con sus capacidades, talentos obtenidos en determinada de modalidad deportiva, con su determinación, dado por la orientación de la especialización deportiva que recibe, así como por la detección que se realice del universo de practicantes, por parte de los encargados de realizar esta función, de que en realidad sean los que más condiciones tengan para su perfeccionamiento.

Esto implica que es primordial tener en cuenta a la selección deportiva como un proceso en el que necesariamente deben involucrarse a la mayor cantidad de practicantes, de manera que se cumpla el derecho de todos como posibles candidatos a ser seleccionados para la práctica de un determinado deporte.

Al respecto, Alexander (2004), señaló que el sector estudiantil cuenta con fortalezas en el amito de acción para seleccionar talentos deportivos, dado el universo de concentración en rangos de edad, lo asequible de la aplicación de los procedimientos de selección, la clasificación y el control de los estudiantes, además de que se cuenta con la disponibilidad de profesionales que una vez preparados pueden comenzar a realizar el proceso de selección. 
En las diferentes carreras que conforman la Escuela Superior Politécnica de Chimborazo $($ ESPOCH) se realizó una observación con la finalidad de estudiar la condición física de los jóvenes que integran las diferentes carreras, haciendo énfasis en el comportamiento de la capacidad de resistencia de los mismos, con vistas, en función de los resultados físicos obtenidos, catalogarlos como un posible talento y evaluar su incorporación a la práctica de un deporte especifico. De ahí que el objetivo general de la investigación se centró en evaluar, en función de resultados relevantes obtenidos en las pruebas de capacidades físicas de resistencia realizadas, la posible elección de talentos deportivos entre los jóvenes universitarios, desde el enfoque del diagnóstico oportuno.

\section{- Evaluación y disposición del ejercicio físico}

En el marco de la Educación Física y el Deporte, existen distintas pruebas y/o test dirigidas a medir las capacidades físicas. Ello nos permitirá conocer la condición física que realmente tienen los practicantes de algún deporte o los individuos en general.

En este ámbito, varios autores, entre los que podemos citar a Castillo (2012), se han referido a los aspectos más evaluados para tener en cuenta en el proceso de selección de talentos, y al respecto han señalado que, las condicionantes de mayor estabilidad que son susceptibles a medir en el proceso de selección de talentos, son los que resultan de factores genéticos, tales como: el VO2 máx., la frecuencia Cardiaca, las pulsaciones por minutos y la frecuencia respiratoria.

Según Morales (2007), para detectar un talento se hace imprescindible la utilización del test de Condición física, que es un examen que ofrece una información respecto al nivel de las capacidades motrices esenciales para tener un determinado rendimiento motor.

Por su parte, Morales, et al. (2007) señalan que el test constituye una herramienta con la que se puede evaluar el desarrollo alcanzado como resultado de la practica sistemática, monitoreada de manera pedagógica y en el que se plasma el desempeño alcanzado de una correcta aplicación de los Programas de Educación Física aplicados.

Desde este punto de vista, se debe considerar la evaluación de la Condición Física como un hecho relacionado al ámbito educativo, y que debe ser utilizado e incluido en los programas de educación física; aspecto razonable si se considera en primer lugar la masa de individuos factibles de evaluar y en segundo término, las posibilidades de obtener distintas informaciones relacionadas a la salud, posibles talentos deportivos, diseño curricular y efectividad de las clases de educación física entre otros aspectos que se podrían investigar y optimizar.

Los resultados que se obtienen de estas evaluaciones nos permitirán saber respecto a cuál capacidad física debemos dirigirnos más y trabajarla con la finalidad de alcanzar una mejora significativa con vistas a lograr nuestro objetivo. En función de ello, debemos escoger dentro de los distintos tipos de evaluaciones, aquella que tengan una mayor fiabilidad, una mayor facilidad de realización y que aporten en mayor grado a las capacidades físicas reales del practicante, según exige nuestra disciplina deportiva. 
Lo anterior es fundamental dado que implica que el entrenamiento de cualquiera de las capacidades biomotoras puede incidir sobre las demás, por lo que estos aspectos son primordiales cuando vayamos a planificar un entrenamiento.

Estos conceptos han perdurado en el tiempo y han sido señalados por diversos autores, entre los que podemos citar Pablos (2005) citando a Kitchin, (1997), quien expresó que, como resultado de un entrenamiento físico adaptado a las necesidades, se hará mucho más tolerable el esfuerzo físico y la posibilidad de una mayor concentración en las orientaciones del ejercicio aplicado.

- Evaluación de la Capacidad Física de Resistencia de los jóvenes universitarios

La evaluación de esta capacidad física fue medida mediante la aplicación del Test de 1000 $\mathrm{m}$, el cual tuvo como objetivo estimar de una forma indirecta la Velocidad Aeróbica Máxima (VAM) y el Consumo Máximo de Oxígeno (Vo:máx).

El mismo contó con los siguientes materiales:

○ Cronómetro

- Pista de atletismo de $1000 \mathrm{~m}$ de longitud.

- Cinta métrica, igual o superior a $50 \mathrm{~m}$.

- Silbato

Indicaciones metodológicas de la prueba física.

- Se verificará la longitud de la pista de atletismo.

- Se verificará la línea de arranque y final de la prueba.

Descripción del ejercicio.

- Posición de arranque de la carrera: Se colocará una pierna al frente ligeramente flexionado, por detrás de la línea de partida y el tronco ligeramente inclinado hacia adelante.

- Desarrollo de la carrera: Desde la línea de arranque, el evaluado partirá corriendo a una velocidad requerida que le permita completar los $1000 \mathrm{~m}$ en el menor tiempo posible. El test concluirá cuando el evaluado logre realizar el completamiento de la distancia, anotándose el tiempo en minutos y segundos que duró la misma.

Valores de Referencia

$\checkmark$ Una vez terminada la carrera y obtenido el tiempo en minutos y segundos realizado, se aplicará la fórmula de Velocidad Aeróbica Máxima (VAM), conforme a la tabla de valores del VAM aprobada por American Heart Association (1972). Exercise Testing and Training of Apparently Healthy Individuals: A Handbook for Physicians.

$\operatorname{VAM}(\mathbf{m} / \mathbf{s})=\mathbf{D} / \mathbf{T}$

(en el caso de $\mathrm{Km} / \mathrm{H}$ usar, VAM(m/s) X 3600/1000)

T- Tiempo convertido en segundos 
D- Distancia (1000 m)

Tabla No 1. Valores de referencia del tiempo en minutos recorrido en $1000 \mathrm{~m}(*)$

\begin{tabular}{lcc}
\hline \multicolumn{1}{c}{ Resultado } & Mujer & Hombre \\
\hline Excelente & $\leq 3: 48$ & $\leq 3: 08$ \\
Bueno & $3: 49-4: 16$ & $3: 09-3: 34$ \\
Medio & $4: 17-4: 58$ & $3: 35-4: 24$ \\
Bajo & $4: 59-5: 54$ & $4: 25-5: 36$ \\
Muy Bajo & $\geq 5: 55$ & $\geq 5: 37$ \\
\hline
\end{tabular}

(*) conforme a American Heart Association (1972). Exercise Testing and Training of Apparently Healthy Individuals: A Handbook for Physicians.

Los resultados obtenidos por cada evaluado o evaluada serán referenciados a la tabla de valores, para conocer en que calificación se enmarca el resultado obtenido.

$\checkmark$ Posteriormente se aplica la fórmula de Volumen Máximo de Oxígeno (VO:Máx), conforme a la tabla de valores del ( $\mathrm{VO}_{2}$ Máx) aprobada por American Heart Association (1972). Exercise Testing and Training of Apparently Healthy Individuals: A Handbook for Physicians.

$\mathrm{VO}_{2} \mathrm{Máx}=438 / \mathrm{T}+3.5(\mathrm{ml} / \mathrm{Kg} / \mathrm{min})$.

$\mathrm{T}=$ Tiempo total recorrido por el evaluado, expresado en minutos.

Tabla No 2. Valores de referencia del $\mathrm{VO}_{2}$ Máx.

\begin{tabular}{lcc}
\hline \multicolumn{1}{c}{ Resultado } & Mujer & Hombre \\
\hline Excelente & $>48$ & $>52$ \\
Bueno & $38-48$ & $43-52$ \\
Medio & $31-37$ & $34-42$ \\
Bajo & $24-30$ & $25-33$ \\
Muy Bajo & $<24$ & $<25$ \\
\hline
\end{tabular}

(*) conforme a American Heart Association (1972). Exercise Testing and Training of Apparently Healthy Individuals: A Handbook for Physicians.

Los resultados obtenidos por cada evaluado o evaluada serán referenciados a la tabla de valores, para conocer en que calificación se enmarca el resultado obtenido.

\section{Metodología}

El tipo de investigación fue descriptiva mediante un enfoque mixto, articulándose elementos cuantitativos y cualitativos, acompañada del empleo de métodos teóricos, tales como: el histórico -lógico, el analítico-sintético, inductivo -deductivo y la observación como método empírico. La muestra fue seleccionada de forma intencional bajo el criterio de inclusión de estar cursando estudios en la Escuela Superior Politécnica de Chimborazo (ESPOCH) en 7 facultades, quedando constituida por 1097 estudiantes. Se trabajó, en el caso que estudiamos, con la aplicación del Test de los 1000 m, el cual permitió evaluar el comportamiento de los evaluados ante la realización de este Test físico, así como las condiciones físicas que pudieran clasificar a alguno de ellos como un talento deportivo, que permita continuar desarrollando su potencial en un deporte especifico. 


\section{Resultados.}

Mediante el indicador de la Capacidad Física de Resistencia, se contó inicialmente con la totalidad de los 1098 estudiantes (femenino: 516 y masculino: 582), que pertenecen a 7 Facultades de la Escuela Superior del Chimborazo, propiciando un abanico de posibilidades de evaluación, en función de las Facultades a las que pertenecen y de su género.

Las Facultades son: Facultad de Ciencias (Ciencias), Facultad de Mecánica (Mecánica), Facultad de Informática y Electrónica (FIE), Facultad de Ciencias Pecuarias (Pecuarias), Facultad de Recursos Naturales (Recursos N), Facultad de Salud Pública y Facultad de Administración de Empresas (FADE).

Tabla No 3. Media de tiempo obtenido en el Test de carrera de $1000 \mathrm{~m}$ por la totalidad de los estudiantes de las distintas Facultades

\begin{tabular}{cc} 
VALORACION DE LA RESISTENCIA MEDIA POR FACULTADES \\
\hline FACULTAD & $\begin{array}{c}\text { Resistencia } \\
\text { A } \mathbf{1 0 0 0} \mathbf{~ m} \\
\text { (en min } \mathbf{~ s e g})\end{array}$ \\
CIENCIAS & 5,42 \\
FADE & 6,09 \\
FIE & 5,97 \\
MECANICA & 4,84 \\
PECUARIAS & 5,77 \\
RECURSOS N & 5,68 \\
SALUD PÚBLICA & 6,02 \\
\hline
\end{tabular}

Una vez realizado el Test de Resistencia a la totalidad de estudiantes de las diferentes facultades, se realizó un análisis del comportamiento del resultado obtenido, en función de la "Tabla: Valores de referencia del tiempo en minutos recorrido en 1000 m", de donde resultó la población de estudiantes que realmente cuentan con las condiciones para conformar el grupo de posibles talentos, la cual ascendió a 221 estudiantes (femenino:43 y masculino:178), con los que se continuaría trabajando para continuar evaluando su desempeño y ulterior desarrollo de posible incorporación a la practica de un deporte especifico.

Para ello, se tomaron los resultados de las calificaciones obtenidas por los estudiantes que conformaron este grupo, enmarcados en calificación catalogada como "Excelente", "Bueno" y "Medio".

Desde el punto de vista individual, se evaluó la cantidad de estudiantes de la totalidad de las facultades, a los que se le aplicó el Test de Resistencia y que obtuvieron resultados de la Velocidad Aeróbica Máxima (VAM), por debajo de los valores de referencia, catalogados con Resultados "Excelente", "Bueno" y "Medio", los que considera este autor, que cuentan con potencialidades para ser considerados como talentos y que se 
favorece realizar un trabajo continuado de entrenamiento, para lograr los resultados deseados.

A continuación, se representa una tabla, en la que aparece el universo de estudiantes, tanto femenino como masculino, que obtuvieron calificaciones que varían entre "Excelentes", "Buenas" y "Medio", por cada Facultad. En la tabla siguiente se observa cual fue el comportamiento por facultades y géneros.

Tabla No. 4: Cantidad de estudiantes femeninos y masculinos que obtuvieron resultados enmarcados en "Excelentes", "Buenos" y "Medio".

\begin{tabular}{lcc}
\hline FACULTADES & FEMENINO & MASCULINO \\
\hline CIENCIAS & 10 & 27 \\
FADE & 15 & 26 \\
FIE & 4 & 31 \\
MECANICA & 0 & 65 \\
PECUARIA & 2 & 8 \\
RECURSOS N & 7 & 14 \\
SALUD PUBLICA & 5 & 7 \\
\hline TOTAL & $\mathbf{4 3}$ & $\mathbf{1 7 8}$ \\
\hline
\end{tabular}

Como puede observarse en la conformación del grupo, del total de féminas inicial, solo el $8,33 \%$ lograron un desempeño para ser incluidas en las categorías planteadas y por su parte el 30,58 \% de los estudiantes masculino lograron por su calificación obtenida, ingresar al grupo.

Tabla No. 5: Media de las calificaciones obtenidas en la carrera de $1000 \mathrm{~m}$.

\begin{tabular}{lccc}
\hline \multicolumn{3}{c}{ MEDIA DE CALIFICACIONES POR FACULTADES Y GENERO (en minutos) } \\
\hline \multicolumn{1}{c}{ FACULTADES } & FEMENINO & MASCULINO & TOTAL \\
INTEGRAL \\
CIENCIAS & 4,38 & 3,83 & 4,10 \\
FADE & 4,23 & 3,66 & 3,94 \\
FIE & 4,52 & 3,85 & 4,19 \\
MECANICA & 0 & 3,90 & 3,90 \\
PECUARIA & 4,46 & 3,83 & 4,15 \\
RECURSOS N & 4,43 & 3,88 & 4,16 \\
SALUD PUBLICA & 4,22 & 4,05 & 4,14 \\
\hline \multicolumn{4}{c}{ TOTAL } \\
\hline
\end{tabular}

Analizando por Facultades se puede apreciar que, en los tiempos obtenidos en la Carrera de Resistencia, en el Total Integral, o sea masculino y femenino de conjunto, solo dos facultades lograron bajar de los 4 minutos, son la Facultad de Mecánica con 3,90 min, siendo la que mejor desempeño obtuvo, y la Facultad FADE con 3.94 min. 
Gráfico No 1: Media de las calificaciones obtenidas en la carrera de $1000 \mathrm{~m}$

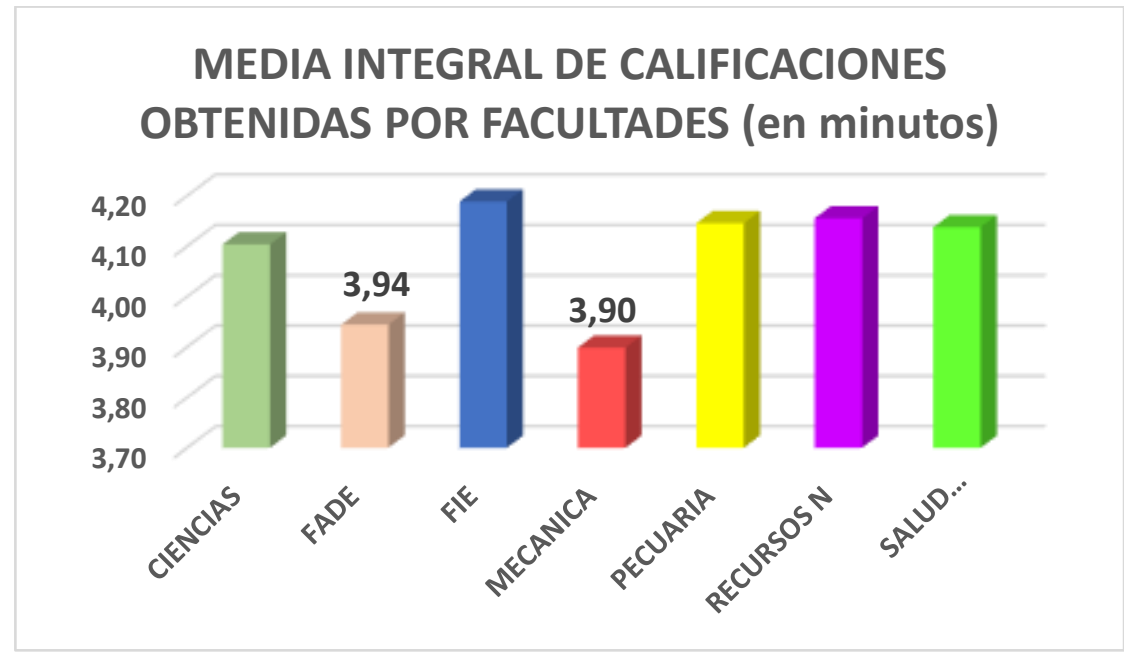

Si lo evaluamos en relación con el desempeño de sus géneros femeninos y masculinos, observamos que, en el caso de los tiempos obtenidos por el género femenino, la media de los tiempos fueron bastantes similares, obteniendo los mejores tiempos, las féminas de la Facultad de Salud Publica con un tiempo de 4,22 min y la Facultad FADE con 4,23 min. Por su parte, los del sexo masculino, tuvieron en la Facultad FADE el mejor desempeño al obtener una media de tiempo de 3,66 min, seguidos de las Facultades de Ciencias y Pecuaria con 3,83 min.

Gráfico No 2: Comportamiento medio de las calificaciones obtenidas por género.
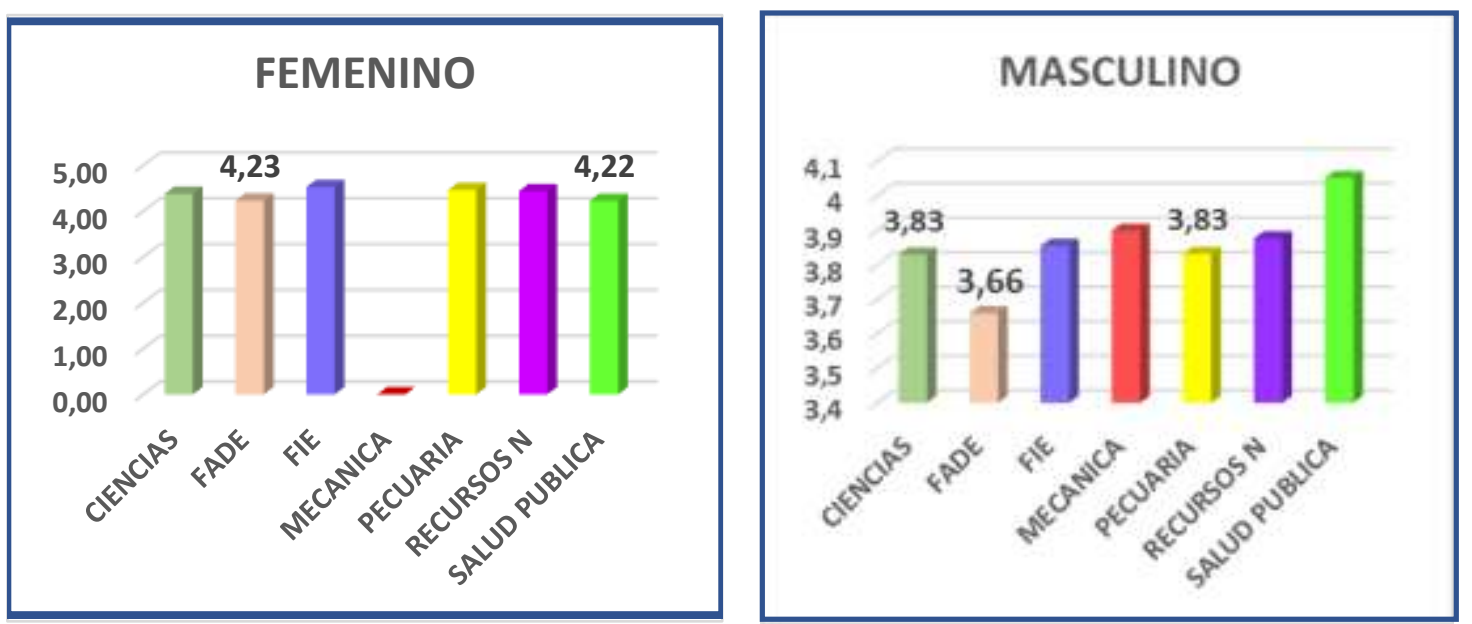

No obstante, realizando una evaluación por categorías, se destaca que entre las féminas que conforman el grupo, cuatro (4) de ellas, o sea el 9,3\% obtienen tiempos catalogados como "Excelentes" dos (2) y otras dos (2) obtienen tiempos catalogados de "Bueno" y a su vez, entre el sexo masculino el 6,2 \% obtuvo tiempos catalogados como "Excelente" $(0,0 \%)$ "Bueno" $(6,2 \%)$. Ninguno de los tiempos obtenidos por los estudiantes del sexo masculino, clasifican como "Excelentes". 
En este orden, provisorio es el desempeño de las 2 féminas que obtuvieron tiempos por debajo del tiempo catalogado como "Excelentes", tales son los casos de una fémina de la Facultad FADE con 3:32 min y la de la Facultad de Salud Pública con 3:41 minutos.

Tabla No 6: Desempeño obtenido por los estudiantes por categoría.

\begin{tabular}{|c|c|c|c|c|c|c|}
\hline \multirow[b]{3}{*}{ FACULTADES } & \multicolumn{6}{|c|}{ EVALUACION DEL DESEMPEÑO OBTENIDO POR CATEGORIAS } \\
\hline & \multicolumn{3}{|c|}{ FEMENINO } & \multicolumn{3}{|c|}{ MASCULINO } \\
\hline & EXCELENTE & BUENO & MEDIO & EXCELENTE & BUENO & MEDIO \\
\hline CIENCIAS & & 1 & 9 & & & 27 \\
\hline FADE & 1 & 1 & 13 & & 3 & 23 \\
\hline FIE & & & 4 & & 4 & 27 \\
\hline MECANICA & & & 0 & & 3 & 62 \\
\hline PECUARIA & & & 2 & & & 8 \\
\hline $\begin{array}{l}\text { RECURSOS N } \\
\text { SALUD }\end{array}$ & & & 7 & & & 14 \\
\hline \multirow[t]{4}{*}{ PUBLICA } & 1 & & 4 & & 1 & 6 \\
\hline & 2 & 2 & 39 & 0 & 11 & 167 \\
\hline & & 43 & & & 178 & \\
\hline & & & 221 & & & \\
\hline
\end{tabular}

A continuación, se presenta el gráfico representativo del desempeño realizado por las féminas en la carrera de $1000 \mathrm{~m}$, en el que se detalla la cantidad de ellas que obtuvieron tiempos catalogados en las diferentes categorías. En el mismo se recoge los dos (2) tiempos catalogados como de "Excelente".

Gráfico No 3. Evaluación del desempeño obtenido por categorías por las féminas.

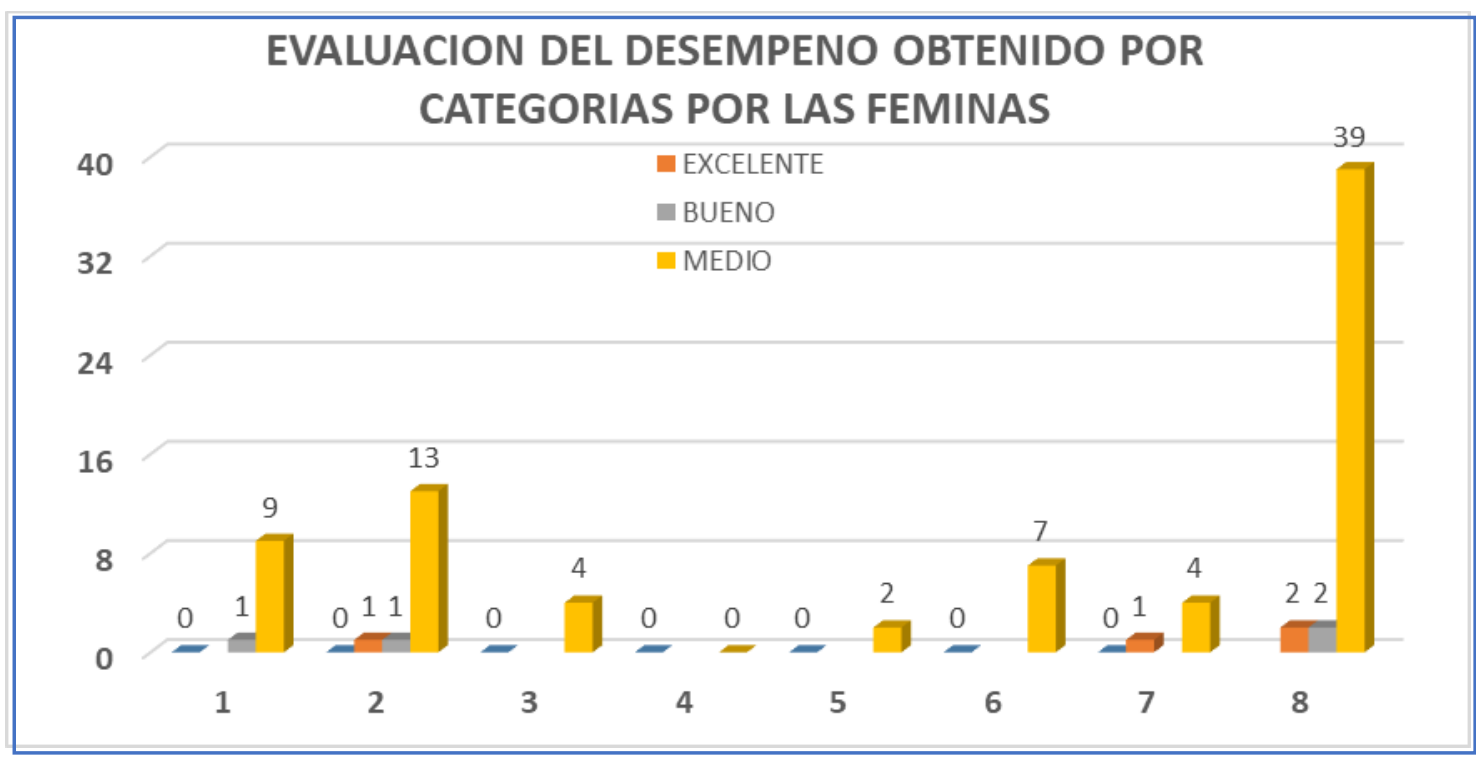

De igual manera se recoge el desempeño realizado por los integrantes del sexo masculino en el Test de carrera de resistencia en los $1000 \mathrm{~m}$, donde se observa que ninguno logró un tiempo con una calificación para ser incluido en la categoría de "Excelente". 
Gráfico No 4. Evaluación del desempeño obtenido por categorías por los estudiantes del sexo masculino.

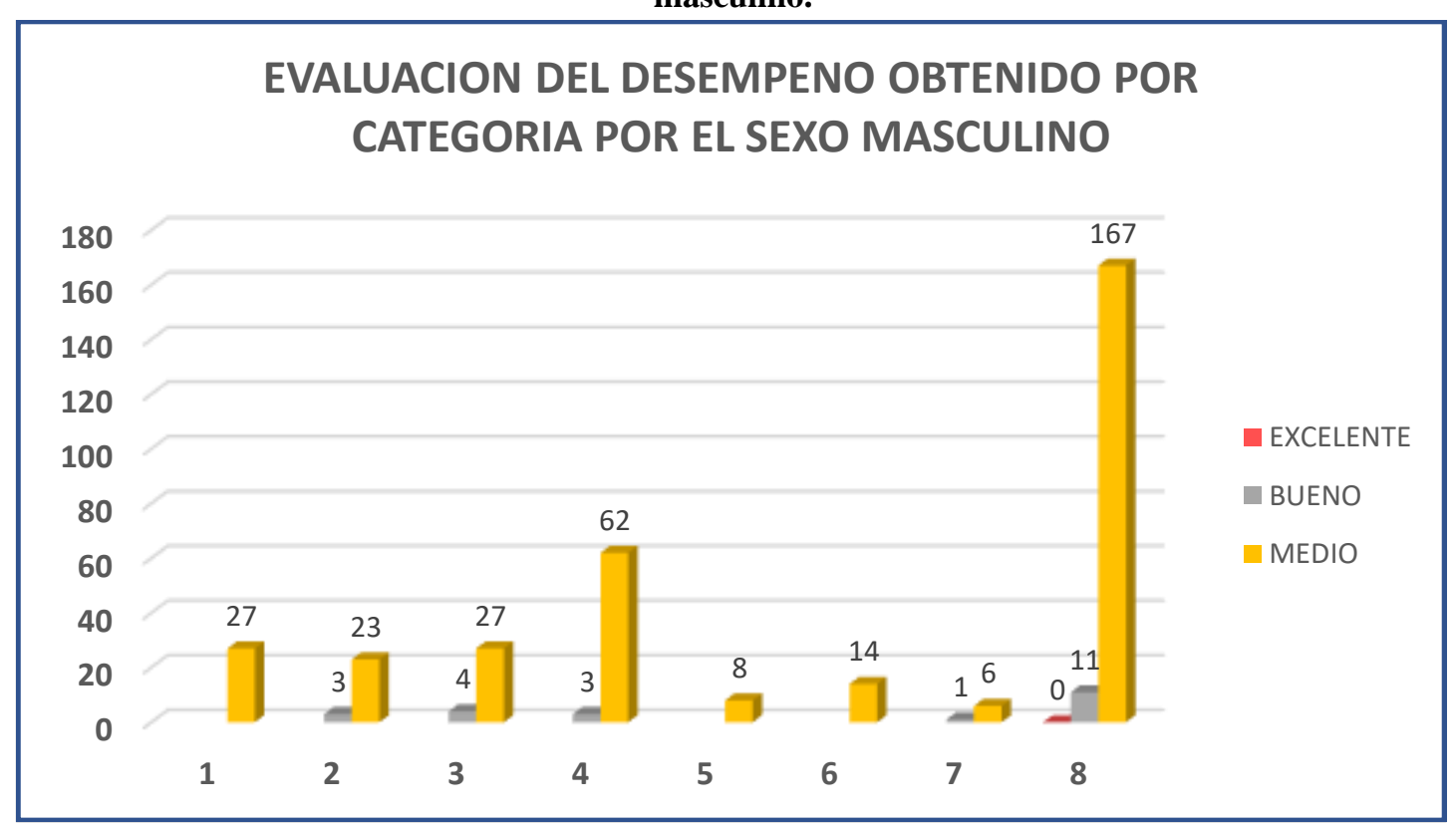

Una vez analizado el resultado obtenido en el Test de los $1000 \mathrm{~m}$ de la carrera de resistencia aplicado a la totalidad del universo de estudiantes, se constató que en función de la fórmula de Velocidad Aeróbica Máxima (VAM), conforme a la tabla de valores del VAM aprobada por American Heart Association (1972), solo el 20,1 \% de ellos realizó tiempos que se incluyeron en las categorías de "Existente", "Bueno" y "Medio", que fue aceptada por el autor para valorar posibles estudiantes que cuentan con desempeños deportivos y características físicas, superiores a la media normal en el desarrollo de una cualidad o capacidad específica, en este caso en la carrera de resistencia en los $1000 \mathrm{~m}$.

Las cualidades detectadas en estos estudiantes pueden ser mejoradas mediante la práctica periódica del ejercicio físico, preferiblemente, conforme han expresado anteriormente varios autores, los cuales deben estar recogidos en programas de Educación Física, que hayan sido elaborados teniendo en cuenta las particularidades de los estudiantes universitarios.

Como ha sido evidenciado los diferentes test de evaluación de las diferentes cualidades físicas ayudan a descubrir los posibles individuos que presentan las condiciones físicas para ser catalogados como talentos. Entre el test utilizado, el Test de los $1000 \mathrm{~m}$ de la capacidad de resistencia, ha sido aplicado por diversos autores, dado que el mismo permite:

1. Aportar un indicador esencial de medición del desempeño de la capacidad física, resistencia de los individuos, en nuestro caso de estudiantes universitarios, lo que nos proveerá el conocimiento respecto a la cantidad de estudiantes que pudieran contar con las condiciones primarias, que permitan ser considerados como talentos, lo que nos permitirá trabajar en su potencial desarrollo deportivo. 
2. Se reafirma la importancia de la utilidad del diagnóstico oportuno, mediante la utilización de Test físicos aplicados a determinada condición física, que nos facilita la posibilidad de obtener de los estudiantes evaluados una cantera para la realización de su desarrollo deportivo posterior.

No obstante, debe tenerse en cuenta que estos test de evaluación de las capacidades físicas, no son suficientes para el trabajo con los estudiantes que pudieran ser seleccionados como talentos, por lo que, como ha sido expresado anteriormente en este artículo, el trabajo no seria efectivo si el mismo no prevé una proyección o transferencia hacia otros aspectos del entrenamiento de la actividad deportiva y de la propia cotidianidad del individuo.

\section{Conclusiones.}

- Diversos autores han coincidido en que entre los aspectos más evaluados para tener en cuenta en el proceso de selección de talentos tenemos los relacionados con los factores genéticos tales como: el VO2 máx., la frecuencia Cardiaca, las pulsaciones por minutos y la frecuencia respiratoria.

- Se demuestra que es esencial considerar la selección deportiva como un proceso en el que necesariamente deben involucrarse la mayor cantidad de practicantes, de forma que permita aplicar los Test de evaluación a la generalidad, cuanto mayor sea la muestra, mayor será la cantidad posibles a ser seleccionados para la práctica de un determinado deporte.

\section{Referencias Bibliográficas.}

American Heart Association (1972). Exercise Testing and Training of Apparently Healthy Individuals: A Handbook for Physicians., Nueva York, Estados Unidos.

Arnett, J. J. (2014). Adolescence and emerging adulthood. A Cultural Aproach. Fifth Edition. Boston, MA: Pearson.

Aránguiz AH, García GV, Rojas DS, Salas BC, Martínez RR, MacMillan KN (2010). Estudio Descriptivo, Comparativo y Correlacional del Estado Nutricional y Condición Cardiorrespiratoria en Estudiantes Universitarios de Chile. Revista Chilena de Nutrición 2010; 37 (1): 70-8.

Castro, M. (2016). Análisis de los parámetros psicosociales, conductuales, físicodeportivos y laborales de los adolescentes de Granada. Tesis Doctoral: Universidad de Granada

Calvo, A., Jiménez, S., Gómez, M., \& Calleja, J., (2013)- Detección y desarrollo del talento deportivo: El estado del arte. Universidad politécnica de Madrid, Facultad de Ciencias de la Actividad Física y del Deporte - INE. 
Cocca, A., Liukkonen, J., Mayorga, D., y Viciana, J. (2014). Healthrelated Physical Activity levels in Spanish youth and young adults. Perceptual and Motor Skills, 118(1), 247-260. doi: 10.2466/10.06.PMS.118k16w1

Castillo, V., (2012). Formación de talentos deportivos en el baloncesto en los niños de 8 a 12 años. Trabajo de Investigación. Universidad de Guayaquil.

Chacón, R., Zurita, F., Castro, M., Espejo, T., Martínez, A. y Pérez, A. J. (2017). Motivational climate in sport and its relationship with digital sedentary leisure habits in university students. Saúde e Sociedade, 26(1), 29-39. Doi: 10.1590/S0104-1290201716656

Chacón, R., Zurita, F., Ubago., JL., González, G., \& Sánchez, M., (2018). Condición física, dieta y ocio digital según práctica de actividad física en estudiantes universitarios de Granada. Revista Euroamericana de Ciencias del Deporte, vol. 7 n. ${ }^{\circ}$ 2, (Supl. 1). ISSN edición web (http://revistas.um.es/sportk): 2340-8812.

Chaparro, D., Ortega, N., \& Romero, S., (2019)- Condición física en adolescentes (resistencia): valores normativos de referencia para la población Bumanguesa 11 a 18 años. Proyecto de grado para optar al título de licenciado en educación física recreación y deporte. Universidad Cooperativa de Colombia Facultad de Educación Programa Licenciatura en Educación Física Recreación y Deporte Bucaramanga.

Delgado, J., Pérez, V., González, F., Suarez, M., (2007)- Evolución de las habilidades motrices y las capacidades físicas en la edad escolar. CEP de Santa Cruz de Tenerife. https://redined.mecd.gob.es > bitstream > handle.

Domínguez, O., Duverger, Y., (2015). Test para la selección de posibles talentos deportivos en el área de medio fondo para ingresar a la categoría juvenil. Lecturas: Educación Física y Deportes, Revista Digital. Buenos Aires, Año 20, No 203. http://www.efdeportes.com/efd203/test-para-la-seleccion-de-talentos-en-mediofondo.htm

Engberg, E., Alen, M., Kukkonen-Harjula, K., Peltonen, J. E., Tikkanen, H. O., y Pekkarinen, H. (2012). Life events and change in leisure time physical activity: A systematic review. Sports Medicine, 42(5), 433-447. doi:10.2165/11597610000000000-00000

García, E., Ortega, F., Ruiz, J., Mesa, J., Delgado, M., González, M. y otros (2007): “El perfil lipídico-metabólico en los adolescentes está más influido por la condición física que por la actividad física (estudio AVENA)", en Revista Española de Cardiología, junio 2007, nº 6, vol. 60, pp. 565-8.

Guimaraes, T., (2002). El entrenamiento deportivo. Capacidades Físicas. San José, C.R.: Universidad Estatal a Distancia (EUNED). 
Han, J. L., Dinger, M. K., Hull, H. R., Randall, N. B., Heesch, K. C., y Fields, D. A. (2008). Changes in women's physical activity during the transition to college. American Journal of Health Education, 39(4), 194-199. DOI: 10.1080/19325037.2008.10599038.

Jaimes, B., Martínez, M., Jerez, J., y Angarita, M., (2019)- Resistencia, fuerza, velocidad e índice de masa corporal (IMC) y su impacto en el rendimiento deportivo de jugadores de baloncesto categoría sub 17. Universidad Cooperativa de Colombia. Licenciatura en Educación Física, Recreación y Deportes. Bucaramanga. Colombia.

Keller, S., Maddock, J. E., Hannöver, W., Thyrian, J. R., y Basler, H. D. (2007). Multiple health risk behaviors in German first year university students. Preventive Medicine, 46(3), 189-195. doi: 10.1016/j.ypmed.2007.09.008.

Martínez, J., Contreras, O., Lera, A., y Aznar, S. (2012). Niveles de actividad física medido con acelerómetro en alumnos de $3^{\circ}$ ciclo de Educación Primaria: actividad física diaria y sesiones de Educación Física. Revista de Psicología del Deporte, 21(1), 117-123.

Martínez, E., (2001). La evaluación Informatizada en la educación Física. Editorial Paidotribo. Primera Edición. Barcelona. España.

Martínez, E., (2002). Pruebas de Aptitud Física. Editorial Paidotribo. Primera Edición. ISBN: 84-8019-641-6. Barcelona. España

Morales, S., \& González, S. A. (2014). Teoría y metodología de la educación física. Quito, Ecuador: Editorial de la Universidad de las Fuerzas Armadas ESPE

Morales, A., (2007). Propuesta de un sistema de normas de rendimiento motor para evaluar la Condición Física y detectar Talentos Deportivos. EFDeportes.com. Revista Digital. Buenos Aires, 112. http://www.efdeportes.com/efd112/talentos.htm.

Morales, L., Pila, H., \& Fleitas, I., (2007)-Normas para evaluar talentos en condición física. Una experiencia en escolares entre 7 y 17 años en el Estado Portuguesa, Venezuela. Segunda Parte.

O’Connor, M., Sanson, A., Hawkins, M. T., Letcher, P., Toumbourou, J. W., Smart, D., y Olsson, C. A. (2011). Predictors of positive development in emerging adulthood. Journal of Youth and Adolescence, 40(7), 860-874. Doi: 10.1007/s10964-0109593-7.

Organización Mundial de la Salud [OMS]. (2010). Recomendaciones mundiales sobre la actividad física para la salud. 
Consultado en la World Wide Web el 5 de junio de 2021:https://www.who.int > factsheet_recommendations.

Ortega, F., Ruiz, M., Castillo, M.J., Moreno, L.A., González-Gross, M., Wärnberg, J. y Gutiérrez, A., (2005): "Bajo nivel de forma física en los adolescentes españoles. Importancia para la salud cardiovascular futura 59 (Estudio AVENA)”, en Revista Española de Cardiología, № 8, vol. 8, agosto 2005, pp. 898-909.

Paucar, Y., (2016)- Indicadores físicos y antropométricos y su incidencia en la detección de talentos deportivos en la provincia de Bolívar. Trabajo de Titulación. Universidad Técnica de Ambato. Ecuador.

Pablos, A., (2005). Valoración de las capacidades físicas y cognitivas en corredores de orientación de la categoría hombres-élite. Universitat de València. Recuperado de https://www.tdx.cat/bitstream/handle/10803/9707/pablos.pdf?sequence=1

Práxedes, A., Sevil, J., Moreno, A., Del Villar, F., \& García, L., (2016). Niveles de actividad física en estudiantes universitarios: Diferencias en función del género, la edad y los estados de cambio. Revista Iberoamericana de Psicología del Ejercicio y el Deporte 2016, 11(1).

Raustorp, A., y Ekroth, Y., (2013). Tracking of pedometerdetermined physical activity: A 10-year follow-up study from adolescence to adulthood in Sweden. Journal of Physical Activity and Health, 10(8), 1186-1192.

Rodríguez F, Cristi C, Villa E, Solis P, Chillon P. (2018). Comparación de los niveles de actividad física durante la vida universitaria. Rev Med Chile 2018; 146: 442-450.

Sáez F \& Gutiérrez A (2007). Los contenidos de las Capacidades Condicionales en la Educación Física. Revista de Investigación en Educación, Nro 4, 2007, pp. 36-60. ISSN: $1697-5200$.

Shephard, R.J., \& Astrand, P.O., (2007). La resistencia en el deporte. Barcelona, España: Paidotribo

Torres, Á. F., Munive, J. E., Alberca, W. V., Díaz, M. G., Ángulo, J. R., \& Morales, S. C. (2017). Adaptaciones curriculares en la enseñanza para alumnos con problemas respiratorios. Revista Cubana de Medicina General Integral, 36(4), 1-19. Recuperado de: http://www.revmgi.sld.cu/index.php/mgi/article/view/717/167.

Vaca, M., Gómez, R., Cosme, F., Mena, F., Yandún, S., Realpe, Z., (2017). Estudio comparativo de las capacidades físicas del adulto mayor: rango etario vs actividad física. Rev Cubana Invest Bioméd vol.36 no.1 Ciudad de la Habana ene.mar. 2017. versión impresa ISSN 0864-0300versión On-line ISSN 1561-3011

Van Dyck D, De Bourdeaudhuij I., Deliens, T., Deforche, B., (2015). Can changes in psychosocial factors and residency explain the decrease in physical activity during 
the transition from high school to college or university? International journal of behavioral medicine 2015; 22 (2): 178-86.

Vinuesa M, Vinuesa I (2016). Conceptos y métodos para el entrenamiento físico. ISBN: 978-84-9091-162-4 (edición libro-e). Catálogo general de publicaciones oficiales http://publicacionesoficiales.boe.es-España.

Weineck J. (2005) Libro: Entrenamiento Total. Editorial Paidotribo. Primera edición: ISBN: 84-8019-805-2. http: //www.paidotribo.com/ Barcelona - España

Weineck, J., (2005). Entrenamiento Total. Editorial paidotribo. (p.131). Primera edición. ISBN: 84-8019-805-2. Barcelona. España.

Zea, A. C., Rodríguez, Y., Correa, J. F., \& Correa, J. C., (2018). Relación entre el porcentaje de grasa y las capacidades físicas básicas en estudiantes universitarios. Rev. colomb. rehabil, 17(2), 82-92. Recuperado de: https://pesquisa.bvsalud.org/portal/resource/pt/biblio-967392.

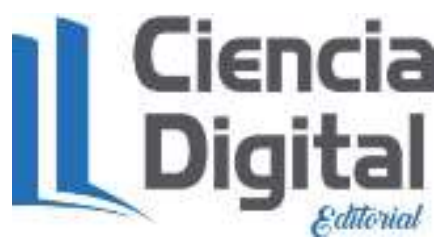




\section{PARA CITAR EL ARTÍCULO INDEXADO.}

Sánchez Espinoza, O. F. ., Gustavo Díaz, L., \& Tocto Lobato, J. G. (2021). La capacidad condicional de resistencia: Su relación con la selección de talentos deportivos en la ESPOCH. AlfaPublicaciones, 3(3.1), 304-324. https://doi.org/10.33262/ap.v3i3.1.98

\section{LCiencia}

El artículo que se publica es de exclusiva responsabilidad de los autores y no necesariamente reflejan el pensamiento de la Revista Alfa Publicaciones.

El artículo queda en propiedad de la revista y, por tanto, su publicación parcial y/o total en otro medio tiene que ser autorizado por el director de la Revista Alfa Publicaciones.
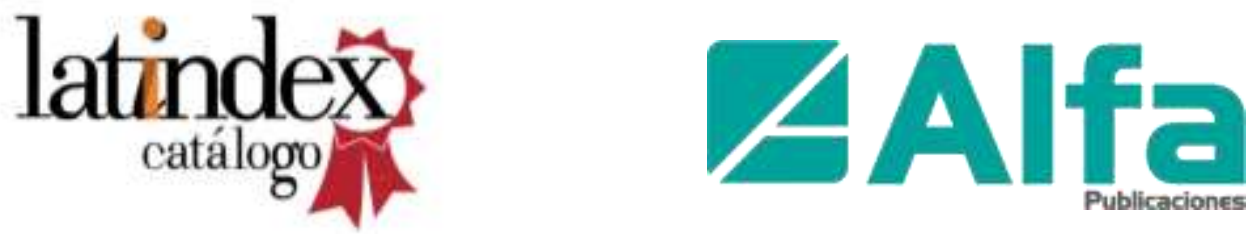